\title{
Agentes Comunitários de Saúde e a promoção do uso racional de medicamentos no nordeste do Brasil
}

Community Health Agents and the promotion of correct use of medication in northeast of Brazil

\author{
T. C. Marques ${ }^{1}$; D. C. S. A. Araujo ${ }^{1}$; A. R. Gama²; G. C. Brito²; R. G. Aguiar ${ }^{3}$; E. \\ M. S. Jesus ${ }^{2}$; A. A. Carvalho ${ }^{2}$; N. A. Silveira ${ }^{3}$; L. L. A. Macêdo ${ }^{4}$; D. P. Lyra- \\ Junior $^{1 *}$ \\ ${ }^{1}$ Laboratório de Ensino e Pesquisa em Farmácia Social (LEPFS), Universidade Federal de Sergipe, 49100-000, \\ Aracaju-SE, Brasil \\ ${ }^{2}$ Departamento de Farmácia, Universidade Federal de Sergipe, 49400-000, Lagarto-SE, Brasil \\ ${ }^{3}$ Instituto de Ciências da Motricidade, Universidade Federal de Alfenas, 37133-840, Alfenas-MG, Brasil \\ ${ }^{4}$ Departamento de Farmácia, Universidade Federal da Paraíba, 58051-900, João Pessoa-PB, Brasil
}

*lepfs.ufs@gmail.com

(Recebido em 22 de janeiro de 2020; aceito em 02 de setembro de 2020)

\begin{abstract}
Os agentes comunitários de saúde (ACS) são importantes atores no processo saúde-doença da comunidade. Este trabalho objetivou descrever o papel dos ACS na promoção do uso racional de medicamentos. Foi realizado um estudo exploratório em três municípios do Nordeste brasileiro entre os anos de 2013 a 2017. A coleta de dados foi realizada por meio de questionários autoaplicáveis. Participaram do estudo 267 ACS; os idosos foram os usuários que mais solicitam informações sobre medicamentos aos ACS (84\%); as principais dúvidas dos usuários estiveram relacionadas à frequência/horário de uso dos medicamentos (86\%); a automedicação foi considerada a principal situação de risco identificada pelos ACS (86\%); 84\% dos ACS referiram não ter conhecimentos suficientes para dar informações sobre medicamentos e apenas 14\% afirmaram ter recebido treinamento com essa temática. Embora os ACS frequentemente identifiquem situações de risco envolvendo o uso de medicamentos, não se sentem seguros para orientar a comunidade. Palavras-chave: Agentes Comunitários de Saúde, atenção primária à saúde, uso de medicamentos.
\end{abstract}

Community health agents (CHAs) are important actors in the community's health-disease process. This study aimed to describe CHAs guidance activities to the population for the correct use of medications. An exploratory survey study was conducted in three Brazilian cities from 2013 to 2017. The data collection involved the application of questionnaires. 267 CHA participated in the study; older people were those who ask the most for medicine therapy information ( $84 \%)$; the main doubts presented by the patients were about medication administration time (86\%); self-medication were pointed out as main risks situations identified by ACS (86\%); $84 \%$ of ACS reported that did not have enough knowledge to give information about medications and $14 \%$ of ACS get trained on this subject. Although CHAs frequently identified risks situations related to the use of medications, they do not feel confident to inform the correct use of medications to community.

Keywords: Community Health Workers, primary health care, drug utilization.

\section{INTRODUÇÃO}

Segundo dados da Organização Mundial da Saúde (OMS), entre 25\% e 70\% dos gastos em saúde nos países em desenvolvimento correspondem a medicamentos e $50 \%$ de todos os medicamentos são prescritos, dispensados ou usados inadequadamente [1]. No Brasil, embora os dados de morbimortalidade relacionados ao uso de medicamentos sejam escassos, revisão sistemática realizada por Souza et al. indicou que sua prevalência provavelmente seja superior a países como os Estados Unidos e Alemanha [2]. Além disso, de acordo com dados do Sistema Nacional de Informações Toxicológicas, o medicamento tem sido descrito como o principal agente causador de intoxicações no Brasil [3].

Incentivar e promover o uso racional de medicamentos tem sido um dos grandes desafios do Sistema Único de Saúde (SUS) e o Ministério da Saúde (MS) enxergou o Agente Comunitário de

$$
094501-1
$$


Saúde (ACS) como um potencial fomentador desta prática. Prova disto foi a publicação da cartilha "O trabalho dos ACS na promoção do uso correto de medicamentos" com o objetivo de repassar a estes profissionais conhecimentos básicos sobre medicamentos [4]. Os ACS são profissionais que atuam para promover o vínculo e a confiança entre as equipes de atenção primária à saúde e a comunidade assistida, assumindo a condição de mediador entre o conhecimento científico e o popular. Neste contexto, pautados pelos princípios da atenção primária à saúde (integralidade, qualidade, equidade e participação social), os ACS estabelecem vínculos com a população e quando devidamente treinados, durante a visita domiciliar, são capazes de realizar esclarecimentos aos usuários sobre o uso correto de medicamentos. Assim, são profissionais aptos a auxiliar no processo de adesão ao tratamento, pois possuem maior contato com a comunidade, conhecem as linguagens, crenças, rotina e nível de entendimento da população [4-7].

Entretanto, apesar da referida Cartilha ter sido publicada há mais de uma década, são escassos os estudos que investiguem o papel e a formação de ACS para promoção do uso racional de medicamentos $[8,9]$. Nesse contexto, o presente estudo objetivou descrever atividades realizadas pelos ACS de três municípios do Nordeste do Brasil para promoção do uso racional de medicamentos.

\section{MATERIAL E MÉTODOS}

Um estudo descritivo, transversal e exploratório foi realizado em três municípios do nordeste brasileiro entre os anos de 2013 e 2017: Lagarto (2013) e Simão Dias (2017), ambos do estado de Sergipe, e Mamanguape (2015) no estado da Paraíba. De acordo com dados do Ministério da Saúde, havia em atividade 194 ACS em Lagarto-SE, 89 em Mamanguape-PB e 99 em Simão Dias-SE nos respectivos anos do estudo, totalizando uma população de 382 ACS. Foram incluídos, por conveniência, os ACS que estivessem presentes nas Unidades Básicas de Saúde durante a visita dos pesquisadores ou em local previamente agendado, sendo excluídos aqueles que não estivessem desenvolvendo atividade laboral durante a realização do estudo.

A coleta de dados foi realizada por meio de um instrumento autoaplicável, previamente desenvolvido e validado por Marques (2008), composto por questões relacionadas a quatro áreas temáticas: 1) Características sociodemográficas (sexo e escolaridade); 2) Abordagem sobre o uso de medicamentos durante as visitas domiciliares; 3) Situações de risco envolvendo o uso de medicamentos pela comunidade; 4) Participação de agentes comunitários em treinamentos específicos acerca do uso de medicamentos. Nos municípios de Mamanguape (PB) e Simão Dias (SE), a aplicação do questionário ocorreu imediatamente antes do início de um treinamento sobre uso racional de medicamentos oferecido pelos pesquisadores. Na cidade de Lagarto (SE), a aplicação se deu por meio da visita dos pesquisadores às Unidades de Saúde em que os ACS estavam vinculados.

Os dados foram analisados por meio da estatística descritiva, utilizando o Programa Microsoft Office Excel 365, com apresentação em frequências relativa e absoluta. O estudo foi aprovado pelo Comitê de Ética em Pesquisa da Universidade Federal da Paraíba (CAAE: 46089315.9.0000.5188) e Universidade Federal de Sergipe (CAAE: 09070112.4.0000.5546). A coleta dos dados foi autorizada pelas Secretarias Municipais de Saúde, o Termo de Consentimento Livre e Esclarecido foi assinado por todos os participantes e o anonimato dos dados foi garantido pelos pesquisadores.

\section{RESULTADOS}

Todos os ACS que estiveram presentes nos treinamentos (Mamanguape-PB e Simão Dias-SE), bem como nas unidades de saúde de Lagarto-SE durante a vista dos pesquisadores, concordaram em participar do estudo. Foram entrevistados 267 ACS, sendo 120 de Lagarto-SE, 79 de Mamanguape-PB e 68 de Simão Dias-SE, correspondendo à 70\% da população estudada. A maioria dos entrevistados era do sexo feminino $(207 ; 78 \%)$ e possuía o ensino médio completo $(147 ; 55 \%)$.

Os Agentes Comunitários de Saúde $(84 ; 31 \%)$ reportaram que foram questionados seis ou mais vezes acerca do uso de medicamentos na semana anterior e que os idosos compuseram o grupo de usuários que mais apresentaram dúvidas acerca desta temática $(225 ; 84 \%)$. As principais 
informações solicitadas pelos usuários aos ACS foram relacionadas à frequência/horário de uso do medicamento $(230 ; 86 \%)$ e duração do tratamento $(160 ; 60 \%)$. Além disso, os ACS referiram identificar situações de risco envolvendo o uso de medicamentos durante as visitas domiciliares, como automedicação $(229 ; 86 \%)$ e horário de administração inadequado $(230 ; 86 \%)$ (Tabela 1).

Tabela 1. Abordagens sobre o uso de medicamentos durante as visitas domiciliares de Agentes Comunitários de Saúde em Lagarto (2013), Mamanguape (2015) e Simão Dias (2017).

\begin{tabular}{|c|c|c|c|c|}
\hline Variáveis & $\begin{array}{c}\text { Lagarto } \\
\text { n [\%] }\end{array}$ & $\begin{array}{c}\text { Mamanguape } \\
n[\%]\end{array}$ & $\begin{array}{c}\text { Simão Dias } \\
\text { n [\%] }\end{array}$ & $\begin{array}{l}\text { Total } \\
\text { n [\%] }\end{array}$ \\
\hline \multicolumn{5}{|c|}{$\begin{array}{l}\text { Com que frequência o Agente Comunitário de Saúde sabe responder a perguntas sobre } \\
\text { medicamentos? }\end{array}$} \\
\hline Sempre & $11[9]$ & $2[2]$ & $4[6]$ & $17[6]$ \\
\hline Quase sempre & 41 [34] & 29 [37] & 24 [35] & 94 [35] \\
\hline Às vezes & $62[52]$ & $41[52]$ & $34[50]$ & 137 [51] \\
\hline Raramente & $5[4]$ & $5[6]$ & 5 [7] & 15 [6] \\
\hline Nunca & $1[1]$ & $2[3]$ & $1[1]$ & $4[1]$ \\
\hline \multicolumn{5}{|c|}{ Frequência com que o ACS foi questionado sobre medicamentos na última semana } \\
\hline Nenhuma & $18[15]$ & $12[15]$ & $10[15]$ & $40[15]$ \\
\hline $1 \mathrm{vez}$ & $5[4]$ & $11[14]$ & $5[7]$ & $21[8]$ \\
\hline 2 ou 3 vezes & $34[28]$ & $30[38]$ & $17[25]$ & $81[30]$ \\
\hline 4 ou 5 vezes & $16[13]$ & $6[8]$ & $19[28]$ & $41[15]$ \\
\hline 6 ou mais vezes & $47[39]$ & $20[25]$ & $17[25]$ & $84[31]$ \\
\hline \multicolumn{5}{|c|}{ Usuários que solicitam informações sobre o uso de medicamentos } \\
\hline Idosos & $98[82]$ & $67[85]$ & $60[88]$ & $225[84]$ \\
\hline Cuidadores de crianças & $96[80]$ & $48[60]$ & $14[21]$ & $158[59]$ \\
\hline Cuidadores de usuários acamados & $57[48]$ & $47[60]$ & $19[28]$ & $153[57]$ \\
\hline Usuárias de contraceptivos & $68[57]$ & $49[62]$ & $25[37]$ & $142[53]$ \\
\hline Cuidadores de usuários de saúde mental & $64[53]$ & $41[52]$ & $18[26]$ & $123[46]$ \\
\hline Gestantes e lactantes & $70[58]$ & - & $32[47]$ & $102[38]$ \\
\hline \multicolumn{5}{|c|}{ Informações sobre o uso de medicamentos solicitadas pelos usuários } \\
\hline Indicação terapêutica & $87[72]$ & $40[50]$ & $29[43]$ & $156[58]$ \\
\hline Dose & $41[35]$ & $38[48]$ & 9 [13] & $88[33]$ \\
\hline Via de administração de medicamentos & $67[56]$ & $38[48]$ & $4[6]$ & $109[41]$ \\
\hline Frequência/horário de uso & $109[91]$ & $65[82]$ & $56[82]$ & $230[86]$ \\
\hline Duração do tratamento & 86 [72] & $48[61]$ & $26[38]$ & $160[60]$ \\
\hline Uso de bebidas alcoólicas durante o & $62[52]$ & $36[46]$ & 33 [49] & $131[49]$ \\
\hline tratamento & $68[57]$ & 23 [29] & 20 [29] & $111[42]$ \\
\hline \multirow{2}{*}{\multicolumn{5}{|c|}{$\begin{array}{l}\text { Efeitos adversos } \\
\text { Situações de risco envolvendo o uso de medicamentos identificadas por Agentes Comunitários de } \\
\text { Saúde }\end{array}$}} \\
\hline & & & & \\
\hline \multirow{7}{*}{$\begin{array}{l}\text { Automedicação } \\
\text { Horário de administração inadequado } \\
\text { Não adesão à farmacoterapia } \\
\text { Dose diferente da prescrita } \\
\text { Medicamento vencido } \\
\text { Medicamento armazenado em local } \\
\text { inadequado } \\
\text { Reação adversa a medicamento }\end{array}$} & 114 [95] & 61 [77] & 54 [79] & $229[86]$ \\
\hline & 108 [90] & $67[85]$ & $55[81]$ & $230[86]$ \\
\hline & 98 [82] & 31 [39] & 27 [40] & 156 [58] \\
\hline & $93[78]$ & $38[48]$ & $26[38]$ & $157[59]$ \\
\hline & $80[67]$ & $32[41]$ & $25[37]$ & $137[51]$ \\
\hline & 88 [73] & 29 [37] & 17 [25] & $134[50]$ \\
\hline & $62[52]$ & $24[30]$ & $10[15]$ & $96[36]$ \\
\hline
\end{tabular}

A maioria dos ACS $(137 ; 51 \%)$ informou que às vezes sabe responder as perguntas dos usuários e 224 ACS (84\%) referiram não ter conhecimentos suficientes para dar informações sobre medicamentos (Tabela 1). Além disso, segundo 203 ACS (75\%), não foram realizadas discussões acerca do uso de medicamentos nas reuniões da equipe de saúde no mês anterior e apenas 28 ACS $(10 \%)$ afirmaram ter recebido treinamento acerca desta temática, enquanto 250 (94\%) sentiam a necessidade de realizá-lo (Tabela 2). 
Tabela 2. Formação de Agentes Comunitários de Saúde para promoção do uso racional de medicamentos em Lagarto (2013), Mamanguape (2015) e Simão Dias (2017).

\begin{tabular}{lcccc}
\hline Variáveis & $\begin{array}{c}\text { Lagarto } \\
\mathbf{n}[\%]\end{array}$ & $\begin{array}{c}\text { Mamanguape } \\
\mathbf{n}[\%]\end{array}$ & $\begin{array}{c}\text { Simão Dias } \\
\mathbf{n}[\%]\end{array}$ & $\begin{array}{c}\text { Total } \\
\mathbf{n}[\%]\end{array}$ \\
\hline Com que frequência a equipe de saúde discutiu o uso de medicamentos com o Agente Comunitário \\
de Saúde nas reuniões de equipe no último mês?
\end{tabular}

O Agente Comunitário de Saúde considera ter conhecimentos suficientes para dar informações sobre medicamentos?

\begin{tabular}{lcccc} 
Sim & $14[12]$ & $4[5]$ & $11[16]$ & $29[11]$ \\
Não & $106[88]$ & $71[90]$ & $47[69]$ & $224[84]$ \\
Não sabe & - & $4[5]$ & $10[15]$ & $14[5]$ \\
\hline O Agente Comunitário de Saúde recebeu algum treinamento acerca do uso de medicamentos? \\
\hline Sim & $3[2]$ & $10[13]$ & $15[22]$ & $28[10]$ \\
Não & $115[96]$ & $66[83]$ & $50[74]$ & $231[87]$ \\
Não sabe & $2[2]$ & $3[4]$ & $3[4]$ & $8[3]$
\end{tabular}

O Agente Comunitário de Saúde sente a necessidade de receber treinamentos acerca do uso de medicamentos?

\begin{tabular}{lcccc} 
Sim & $116[97]$ & $76[96]$ & $58[85]$ & $250[94]$ \\
Não & $4[3]$ & $3[4]$ & $10[15]$ & $17[6]$ \\
\hline
\end{tabular}

\section{DISCUSSÃO}

A predominância do sexo feminino e do ensino médio completo é semelhante ao perfil identificados em estudos de diferentes regiões do Brasil [10-13]. O trabalho do ACS, historicamente, tem apresentado inclinação para o cuidado, papel geralmente desempenhado pela mulher, o que tem lhe conferido maior credibilidade e sensibilidade com a comunidade $[14,15]$. Embora a Lei 11.350/2006, em vigor durante a realização deste estudo, exigisse, no mínimo, o ensino fundamental completo como requisito, a adoção de avaliações cognitivas no processo seletivo do ACS tem contribuído para o aumento do nível de escolaridade destes profissionais, pois privilegia aqueles com mais anos de estudo [16]. Atualmente, com a publicação da Lei ${ }^{\circ} 13.595$, de 05 de janeiro de 2018, a escolaridade mínima requerida passou a ser o ensino médio completo [17].

Os idosos frequentemente têm questionado os ACS acerca do uso de medicamentos. Isto pode estar relacionado ao crescente envelhecimento populacional e aumento da prevalência de doenças crônicas não transmissíveis, que tem contribuído para que os idosos se tornem potenciais usuários de serviços de saúde e de medicamentos [18, 19]. Estudo realizado por Leite et al. (2016) [20] constatou que a prática de ACS junto à população idosa é permeada por dificuldades em virtude das limitações ou resistências apresentadas pelo idoso. Dentre as barreiras identificadas, destaca-se a baixa capacidade de assimilação das orientações, geralmente em função da reduzida escolaridade, o que dificulta a adesão à terapia medicamentosa. Em contrapartida, Remondi et al. (2014) [21] identificaram que o envolvimento do ACS tem impacto positivo na adesão ao tratamento por usuários de medicamentos para doenças crônicas. Ainda que o ACS não desenvolva atividades específicas para o monitoramento da terapia, ele pode atuar sanando dúvidas e preocupações que poderiam interferir na adesão.

A adesão ao tratamento também pode ser influenciada pelo conhecimento do usuário acerca da farmacoterapia prescrita. Dúvidas quanto a frequência, horário de uso e duração do tratamento pode estar relacionada a dificuldades no fornecimento de informações pelos profissionais de saúde, como o uso de termos técnicos, ou pelo constrangimento dos usuários em expressarem dúvidas [22]. Em estudo realizado por Kauling et al. (2013) [23], os ACS reportaram que as principais situações de 
risco identificadas foram o armazenamento inadequado, automedicação, posologia incorreta, reações adversas, falta de compreensão e adesão à farmacoterapia e o uso de medicamentos associados a plantas medicinais.

Apesar das dúvidas de usuários e situações de risco envolvendo o uso de medicamentos serem comuns no cotidiano do ACS, a maioria acredita não ter conhecimentos suficientes e sente necessidade de treinamentos e informações adicionais para orientar a comunidade adequadamente. Apesar disso, aspectos relacionados à utilização de medicamentos não tem sido um tema discutido nas reuniões da equipe de saúde. Dessa forma, ausência destes treinamentos e discussões pode fragilizar o processo de orientação e contribuir para práticas profissionais inadequadas [24, 25]. Nesse contexto, é importante ressaltar que discussões sobre promoção do uso racional de medicamentos no território devem ser incentivadas durante as reuniões da equipe de saúde. Além disso, existe a necessidade de a equipe ser apoiada por uma rede multiprofissional, que reúna os conhecimentos de diversos profissionais.

Este estudo apresenta algumas limitações. Apesar de ser um estudo em três municípios do Nordeste, não é possível extrapolar os resultados porque a amostra não é representativa da região. Por outro lado, a realização do estudo em cidades amplia a amostra estudada e permite identificar possíveis diferenças e semelhanças dentro de uma mesma região geográfica.

\section{CONCLUSÃO}

A partir dos dados deste estudo, foi possível concluir que os ACS durante as visitas domiciliares identificam situações de risco relacionadas ao uso de medicamentos e são frequentemente questionados pelos usuários sobre a farmacoterapia. Pacientes idosos são os usuários que mais solicitam informações sobre terapia medicamentosa e a automedicação é a situação de risco mais identificada durante suas visitas domiciliares. No entanto, poucos ACS participaram de treinamentos sobre o uso de medicamentos. Dessa forma, os ACS devem ser treinados para identificar situações de risco em relação à farmacoterapia e comunicar à equipe de saúde para o planejamento das intervenções adequadas. Além disso, há a necessidade de introduzir treinamento teórico e prático para melhorar a orientação da comunidade e incorporar habilidades e competências específicas para este fim pelos ACS.

\section{REFERÊNCIAS BIBLIOGRÁFICAS}

1. World Health Organization. Quality Assurance and Safety of Medicines Team. The safety of medicines in public health programmes: pharmacovigilance, an essential tool. Geneva: WHO; 2006. 60p.

2. Souza TT, Godoy RR, Rotta I, Pontarolo R, Fernandez-Llimos F, Correr CJ. Morbidade e mortalidade relacionadas a medicamentos no Brasil: revisão sistemática de estudos observacionais. Rev Ciênc Farm Básica Apl. 2015;35(4): 519-532.

3. Nunes CRM, Alencar GO, Bezerra CA, Barreto MDFR, Saraiva EMS. Panoramas das Intoxicações por Medicamentos no Brasil. Revista E-Ciência. 2017;5(2): 98-103, doi: 10.19095/rec.v5i2.247

4. Brasil. Ministério da Saúde. O trabalho dos Agentes Comunitários de Saúde na promoção do uso correto de medicamentos. 2 ed. Brasília: Ministério da Saúde; 2006.72 p.

5. Miasso AI. Terapêutica Medicamentosa: orientação e conhecimento do paciente na alta e pós-alta hospitalar [dissertação]. São Paulo (SP): Universidade de São Paulo; 2002. 118 p.

6. Oliveira EM, Spiri WC. Programa Saúde da Família: a experiência de equipe multiprofissional. Rev Saúde Pública. 2006;40(4):727-733.

7. Marodin G, Merotto J, Zanchet F, Boeno DC, Fernandes A, Cervi MC. Agentes comunitários de saúde, idosos e PET-Saúde: Uso e Cuidados com Medicamentos. Revista Contexto \& Saúde. 2011;11(20): 635-642, doi: 10.21527/2176-7114.2011.20.635-642.

8. Marques TC. As atividades de agentes comunitários de saúde e a promoção do uso correto de medicamentos em unidades do Distrito de Saúde Oeste de Ribeirão Preto - SP [dissertação]. Ribeirão Preto (SP): Universidade de São Paulo, 2008. 105 p.

9. Guimarães MSA, Tavares NUL, Naves JDOS, Sousa MFD. Family health strategy and the rational use of medications: work of the community agents of the Municipality of Palmas (TO). Trab Educ Saúde. 2017;15(1):183-203, doi: 10.1590/1981-7746-sol00037 
10. Manoel AL, Rodrigues AB, Piva EZ, Warpechowski TP, Schuelter-Trevisol F. Avaliação do conhecimento sobre o vírus do papiloma humano (HPV) e sua vacinação entre agentes comunitários de saúde na cidade de Tubarão, Santa Catarina, em 2014. Epidemiol Serv Saúde. 2017; 26:399-404, doi: $10.5123 / \mathrm{s} 1679-49742017000200017$

11. Mascarenhas CHM, Prado FO, Fernandes MH. Fatores associados à qualidade de vida de agentes comunitários de saúde. Ciênc Saúde Colet. 2013;18(5):1375-1386.

12. Ramdohr Sobrinho EC, Freitas KG, Figueiredo RM, Calliari JS. A tuberculose na estratégia de saúde da família: o conhecimento dos agentes comunitários de saúde. Rev Eletr Enf [Internet]. 2013 AbrJun;15(2):416-421, doi: 10.5216/ree.v15i2.16982.

13. Cazola LHO, Tamaki EM, Pontes ERJC, Andrade SMO. Incorporação das atividades de controle da dengue pelo agente comunitário de saúde. Rev Saúde Pública. 2014;48(1):113-122, doi: 10.1590/S0034-8910.2014048004687

14. Garcia ACP, Lima RDCD, Galavote HS, Coelho APS, Vieira ECL, Silva RC, Andrade MAC. Agente comunitário de saúde no Espírito Santo: do perfil às atividades desenvolvidas. Trab Educ Saúde. 2017;15(1):283-300, doi: 10.1590/1981-7746-sol00039

15. Mota RRDA, David HMSL. A crescente escolarização do agente comunitário de saúde: uma indução do processo de trabalho? Trab Educ Saúde. 2010;8(2): 229-248, doi: 10.1590/S198177462010000200004

16. Brasil. Casa Civil. Lei 11.350, de 5 de outubro de 2006. Regulamenta o $§ 50$ do art. 198 da Constituição, dispõe sobre o aproveitamento de pessoal amparado pelo parágrafo único do art. 20 da Emenda Constitucional no 51, de 14 de fevereiro de 2006, e dá outras providências. Brasília (DF): Diário Oficial da República Federativa do Brasil, Seção 1; 6 Out 2006.

17. Brasil. Casa Civil. Lei 11.350, de 5 de outubro de 2006. Lei n ${ }^{\circ} 13.595$, de 05 de janeiro de 2018. Altera a Lei $n^{\circ} 11.350$, de 5 de outubro de 2006, para dispor sobre a reformulação das atribuições, a jornada e as condições de trabalho, o grau de formação profissional, os cursos de formação técnica e continuada e a indenização de transporte dos profissionais Agentes Comunitários de Saúde e Agentes de Combate às Endemias. Brasília (DF): Diário Oficial da República Federativa do Brasil, Seção 1:1; 8 Jan 2018.

18. Desevo G, Klootwyk J. Pharmacologic issues in management of chronic disease. Prim Care. 2012 Jun;39(2):345-362, doi: 10.1016/j.pop.2012.03.007

19. Veras R. Envelhecimento populacional contemporâneo: demandas, desafios e inovações. Rev Saúde Pública. 2009;43(3):548-554, doi: 10.1590/S0034-89102009005000025.

20. Leite MT, Dal Pai S, Hildebrandt LM, da Silva LAA. Vivências de agentes comunitários de saúde na atenção a idosos acometidos por doenças crônicas. Rev Rene. 2016 Jul-Ago;17(4):574-584, doi: 10.15253/2175-6783.2016000400019

21. Remondi FA, Cabrera MAS, Souza RKT. Não adesão ao tratamento medicamentoso contínuo: prevalência e determinantes em adultos de 40 anos e mais. Cad. Saúde Pública. 2014 Jan;30(1):126136, doi: 10.1590/0102-311X00092613

22. Kardas P, Lewek P, Matyjaszczyk M. Determinants of patient adherence: a review of systematic reviews. Front Pharmacol. 2013 Jul;(4):91, doi: 10.3389/fphar.2013.00091

23. Kauling GP, Ceretta LB, Schwalm MT, Dagostim VS, Soratto MT. Utilização de medicamentos: limites e possibilidades das orientações dos Agentes Comunitários de Saúde às famílias. O Mundo da Saúde. 2013;37(1):44-55.

24. Nunes CC, Amador TA, Heineck I. O medicamento na rotina de trabalho dos agentes comunitários de saúde da unidade básica de saúde Santa Cecília, em Porto Alegre, RS, Brasil. Saúde Soc. 2008;17(1):8594, doi: 10.1590/S0104-12902008000100008

25. Queiroz DM, Silva MRF, Oliveira LC. Continuing education for community health agents: potentialities of an education guided by the framework of health and popular education. Interface (Botucatu). 2014;18(2):1199-1210, doi: 10.1590/1807-57622013.0303 